Cinémas

Revue d'études cinématographiques

Journal of Film Studies

\title{
L'Oumigmag, ce difficile parcours entre le mot et les choses
}

\section{Françoise Beaulieu}

Volume 5, numéro 3, printemps 1995

Cinélekta 1

URI : https://id.erudit.org/iderudit/1001150ar

DOI : https://doi.org/10.7202/1001150ar

Aller au sommaire du numéro

Éditeur(s)

Cinémas

ISSN

1181-6945 (imprimé)

1705-6500 (numérique)

Découvrir la revue

Citer cet article

Beaulieu, F. (1995). L'Oumigmag, ce difficile parcours entre le mot et les choses. Cinémas, 5(3), 115-130. https://doi.org/10.7202/1001150ar

\section{Résumé de l'article}

Plus que tout autre film depuis la série Au pays de Neufve-France (1959-1960), L’Oumigmag (1993) se présente comme une entreprise de dénomination. Nommer revient à exercer trois fonctions sémiotiques. Désigner, en images ou en paroles, consiste à attirer l'attention sur un objet sans considération de sa signification. La dénotation et la connotation interviennent ensuite pour rassembler des prédicats autour de ce même objet : dans le premier cas, le sens reste le même pour tous les observateurs tandis que dans l'autre, il fluctue en fonction de critères individuels. La stratégie discursive de Pierre Perrault se résume à peu près à ceci : ignorer la dénotation de l'animal pour mettre en relief la désignation et les connotations de son individualité. Réussit-il à démontrer qu'il est possible de nommer l'Oumigmag aussi bien en français qu'en langues vernaculaires? 


\title{
L'Oumigmag, ce difficile parcours entre le mot et les choses
}

\author{
Françoise Beaulieu
}

\section{RÉSUMÉ}

Plus que tout autre film depuis la série $A u$ pays de Neufve-France (1959-1960), L'Oumigmag (1993) se présente comme une entreprise de dénomination. Nommer revient à exercer trois fonctions sémiotiques. Désigner, en images ou en paroles, consiste à attirer l'attention sur un objet sans considération de sa signification. La dénotation et la connotation interviennent ensuite pour rassembler des prédicats autour de ce même objet: dans le premier cas, le sens reste le même pour tous les observateurs tandis que dans l'autre, il fluctue en fonction de critères individuels. La stratégie discursive de Pierre Perrault se résume à peu près à ceci : ignorer la dénotation de l'animal pour mettre en relief la désignation et les connotations de son individualité. Réussit-il à démontrer qu'il est possible de nommer l'Oumigmag aussi bien en français qu'en langues vernaculaires?

\section{ABSTRACT}

More than any other movie, ever since the series $A u$ pays de Neufve-France (1959-1960), L'Oumigmag (1993) is seen as a denomination enterprise. Naming amounts to exercising three semiotic functions. Pointing out, in pictures or in words, consists in drawing attention to an object without any consideration for its meaning. Then, denotation and connotation intervene to gather predicates together around this very object. In the first instance, the meaning remains the same for all observers, whereas in the other it varies according to individual criteria. Pierre Perrault's discursive strategy 
can be summed up as follows: to be unaware of the animal's denotation in order to highlight the designation and connotations of its individuality. Is he successful in demonstrating that it is quite possible to name the Oumigmag, as well in French as in vernacular languages?

Après trente ans de carrière, nous commençons à avoir une bonne idée de la façon de procéder de Pierre Perrault bien qu'il ait encore une fois réussi à nous prendre par surprise avec L'Oumigmag (1993). Dès le début des années soixante-dix, ses films se sont mis à s'écarter des attentes suscitées par Pour la suite du monde (1963) parce que la technique du cinéma direct n'y était plus respectée au pied de la lettre, si elle le fut jamais, et que des côtés plus scabreux de la réalité, aux antipodes du discours emblématique d'un Alexis Tremblay, n'ont pas échappé à la vigilance de la caméra. Pourtant, en raison de la continuité de son œuvre et de la vigueur de l'expression poétique, la réputation du cinéaste n'a cessé de grandir, forçant le public à s'adapter à un objet décidément peu banal.

Pas un seul instant n'a-t-il cessé de rechercher l'authenticité, d'abord en "donnant la parole aux gens" comme il s'est longtemps plu à le dire lui-même, puis en montrant l'altérité des choses qui se dérobent sous les apparences. Voilà la double fonction qu'il attribue à l'objectif documentaire'. Seulement, il faut comprendre que ce dessein n'est pas accompli sans rigueur: pour le cinéaste, il importe de s'opposer aux habitudes d'interprétation, aux lois d'un genre donné, aux modes de réalisation, mais surtout ne pas faire semblant d'être marginal pour défendre une position idéologique. Dans L'Oumigmag, il n'est même plus question de capter l'affleurement d'une différence qui égalerait celle des flamboyants personnages du Retour à la terre (1976), d'Un pays sans bon sens (1970) ou des Voitures d'eau (1968); au contraire, nous sommes conviés à nous familiariser avec une nouvelle forme d'exotisme québécois, celui du bœuf musqué, inaccessible aux masses parce que requérant la médiation d'un code, d'une langue qui bouscule les clichés et l'uniformité des choses. 


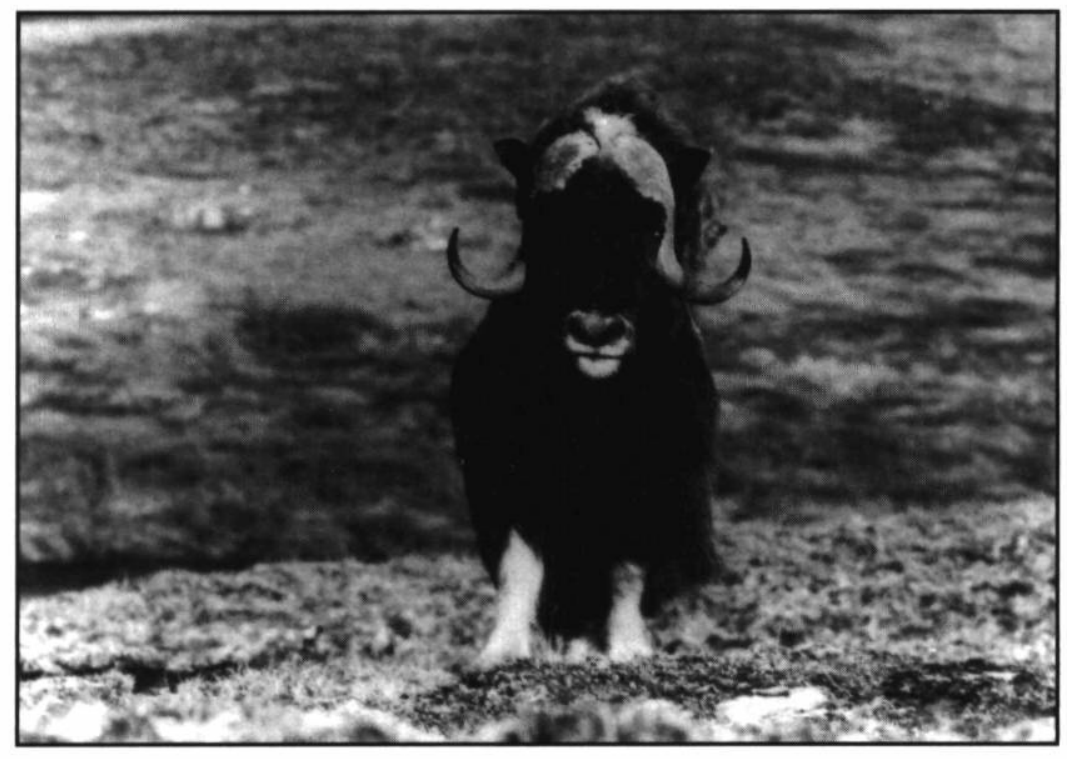

\section{L'Oumigmag de Pierre Perrault (1993)}

Collection ONF

A la lumière des connaissances actuelles, le "chasseur au tambour ", donné par Perrault comme le seul observateur à vraiment connaître la réalité du bœuf musqué, constitue une énigme parce qu'il n'a pas su nous transmettre son nom. Ignorant l'écriture, sa rêverie, à peine esquissée sur les parois des cavernes, est demeurée sans explication et ne porte aucune signature. Apposée comme un sceau, signe de l'appropriation d'une connaissance, celle-ci eût certainement ajouté à la signification de l'image rupestre parce qu'elle a la curieuse propriété d'en absorber les qualités et que sa présence indique une maturité certaine dans l'interprétation des choses. Les phénomènes indifférents, sans doute, ne se méritent jamais d'appellation puisque l'attention ne s'est pas portée suffisamment sur eux; règle générale, $c^{\prime}$ est le cas de ce qui se trouve au nord du $60^{\circ}$ parallèle, à en juger du moins par les traités de botanique et de biologie québécois. L'altérité de la Baie-aux-Feuilles, lexicalisée dans les patois vernaculaires, reste alors ignorée par la majorité des habitants de la vallée du Saint-Laurent et du reste du monde. Or, par le truchement d'un commentaire poétique, d'une sorte de paraphe, 
nous sommes invités à rassembler des prédicats autour de la "description " cinématographique du bœuf musqué, dans l'intention que le nom l'Oumigmag, étrange et anguleux avant le visionnement, s'intègre harmonieusement dans la nomenclature de la langue française.

Parmi tous les films de Pierre Perrault, il n'y en a sans doute aucun qui aborde aussi directement et aussi profondément la délicate question de la dénomination qui, comme nous le savons, a soulevé jusqu'à ce jour énormément de controverse. Depuis le célèbre Cratyle de Platon, premier ouvrage entièrement consacré au nom propre dont nous gardions la mémoire, de nombreux philosophes, historiens, anthropologues, linguistes, littérateurs et théoriciens de toute obédience ont vainement tenté d'en donner une définition universelle, avec pour résultat la mise à jour de doctrines aussi variées que contradictoires.

Socrate et Hermogène, quoi qu'en disent certains commentateurs d'aujourd'hui, n'ont jamais réussi à établir s'il existe un lien naturel ou conventionnel entre le nom et la chose qu'il représente, pas plus que Gottlob Frege ${ }^{2}$ et John Stuart Mill ${ }^{3}$ n'ont pu déterminer s'il se rattache effectivement à un sens. De même, portés par les espoirs de la philosophie analytique et du structuralisme, Bertrand Russell ${ }^{4}$, Ludwig Wittgenstein ${ }^{5}$, Paul Strawson ${ }^{6}$ et John Searle ${ }^{7}$ ont exploré ce qu'ils croyaient être ses trois fonctions canoniques, pour s'apercevoir qu'elles recèlent toutes une énigme résistant et niant à la fois leurs tentatives de formulation. Avec un certain recul, John Algeo ${ }^{8}$, Farhang Zabeeh ${ }^{9}$, Holger Sørensen ${ }^{10}$, Paul Gardiner ${ }^{11}$ et Claude LéviStrauss ${ }^{12}$ ont bien tenté de faire un bilan de la situation, constatant qu'il reste possible, après tant d'efforts de rationalisation, de soutenir à la fois que le nom propre désigne un objet "unique" ou un objet "collectif", qu'il ne dénote "rien" ou qu'il dénote "de façon exhaustive", mais surtout, qu' «il n'a pas de connotation" ou qu'il possède la propriété de "connoter infiniment".

Nommer, par conséquent, prend l'allure d'un procédé linguistique, cinématographique et poétique ambigu. Benjamin Disraeli dans Influence of Names ${ }^{13}$ observait justement que " [...] les effets qu'il produit sur l'imagination sont l'une des illusions les plus extraordinaires du genre humain». Ce témoignage, cor- 
roboré par tant d'autres, nous laisse perplexes quand l'auteur de L'Oumigmag, à maintes reprises ${ }^{14}$, nous suggère qu'il est un impératif de connaissance de la réalité et que de ne pas procéder à la dénomination pour éviter ses inconvénients revient à se priver d'un des moyens d'expression les plus puissants qu'il se puisse concevoir. Aussi convient-il de le suivre dans sa démarche et de voir dans quelle mesure il l'intégrera à un court métrage intitulé L'Objectif documentaire.

Dès le début des années soixante s'amorce avec les films de Perrault de la série $A u$ pays de Neufve-France une entreprise de dénomination qui, sans le déclarer ouvertement, se propose de mettre à jour les particularités spatiales et humaines du pays québécois. Y a-t-il derrière le procédé une "intention" politique ou idéologique? Sans doute, bien que considérés seuls, les " effets pratiques" de l'attribution du nom s'avèrent significatifs. Car on ne nomme pas un être ou une personne d'une manière innocente: de leur rattacher un signe permanent représente une marque d'intérêt indéniable, surtout que tant d'objets de notre environnement, que nous voyons pourtant tous les jours et qui nous sont chers, n'auront jamais ce privilège. De nommer par exemple une montre-bracelet "Robert " ou "Georges " ou "Yvette" paraît certainement ridicule, même si rien en théorie ne nous empêche de le faire. En contrepartie, le fait de ne pas nommer une réalité saillante pour un individu ou un peuple constitue platement une preuve d'indifférence, comme l'ont brillamment soutenu notre cinéma et notre littérature nationale.

L'Oumigmag, n'étant pas sans rappeler les œuvres de Maurice Proulx, Albert Tessier, Marie-Victorin, Jean Rouch, Dziga Vertov et bien d'autres, constitue une nouvelle tentative d'appropriation du réel, un geste qui signifiera davantage pour ceux qui ont une connaissance préalable du Nunavik, c'est-à-dire dans l'ordre l'Amérindien et le chercheur québécois, mais qui vise tout de même l'humanité dans son ensemble. Il s'agit de voir, de comprendre et ultimement de communiquer avec le bœuf musqué afin qu'il devienne possible, pour ainsi dire, de l'appeler par son nom, à l'exemple du chasseur primitif qui était loin de se douter que ses paroles deviendraient un témoignage, une légende et du cinéma documentaire. "L’Oumigmag", vocable connu de ceux 
qui ont tué la bête sous l'impulsion de la faim, de ceux qui savent son incroyable survivance, sa méfiance et la signification de ses cornes, de ceux qui reconnaissent en elle une suspension du temps et de l'espace, est-il en voie de gagner une nouvelle acception tout en consacrant une fois de plus l'échec de la théorie qui tente de le classer? Voyons un peu de quoi il retourne.

Le nom propre possède un maximum de trois fonctions sémiotiques que nous passerons brièvement en revue. La première, la "désignation", consiste à faire usage du nom dans le simple but de mobiliser l'attention, sans que ne soit pris en considération le sens de l'objet visé. Les pronoms démonstratifs " ceci ", "cela ", "celui-ci ", "celui-là ", qui montrent un objet sans en dire quoi que ce soit, illustrent assez bien le procédé, mettant en relief un pouvoir de "monstration" que l'image possède évidemment à un degré plus élevé que les symboles linguistiques. Voilà pourquoi nous porterons d'abord notre attention sur elle, avant que le chasseur d'images ne nous raconte son périple. Or la fonction de désignation a ceci de particulier que même dans un univers fictionnel, elle se doit d'offrir une cohérence minimale, sinon l'interprète n'arriverait pas à la juger vraie ou fausse, faute de la rattacher à des faits empiriques connus et vérifiables. Il va de soi que dans le cadre d'un film documentaire, cette contrainte revêt une importance décisive et constitue la pierre d'assise de toute la réflexion.

Si nos sens déforment la réalité parce que conditionnés par nos désirs et nos connaissances préalables, la pellicule, elle, ne ment pas et s'impose comme la réaction immédiate de la lumière à un moment précis. Contrairement à ce qu'on a pu dire, Pierre Perrault n'est pas dupe de cette objectivité et reconnaît que la sélection d'une image plutôt qu'une autre, le montage, la lumière, la durée, le rythme, le grain, l'orientation, la focale et la synchronisation comptent au nombre des distorsions introduites. À ce sujet, il écrit :

La lumière, elle-même, est-elle vraiment objective? Elle donne à voir sa substance et celle de la laine. Est-ce que l'image regarde la laine ou la lumière? Le vent à son tour intervenant, révélant toutes sortes de souplesse du poil et des crinières. Se jouant de la lumière. Trop 
d'informations peut-être? Où est l'objectivité ? La lumière falsifie, embellit. Magie de la lumière. L'image se rapproche des chevelures. Vent dans les poils. Lumière dans le vent, objet sans objectivité, sans queue ni tête, meule, gerbe. Le poil est presque blanc. La lumière est-elle objective? Elle change la couleur. Elle transforme la bête. Il n'y a plus de bête. Mais de la lumière qui blanchit de la laine. Et quoi encore? Comment dire tout ce que l'image propose?

Mais il reste que cette propriété de fixer le temps et l'espace forme une mémoire empirique que la subjectivité ne peut jamais réduire complètement, surtout quand une volonté ferme s'applique minutieusement à éviter que cela ne se produise. L'image comporte une quantité d'information qui excède largement la capacité d'observation de l'individu et qui, lors de visionnements successifs, lui réserve des surprises. En y regardant à répétition, il finira cependant par convenir que sa première impression comportait des erreurs et des lacunes, imperceptibles désormais parce que l'esprit, quelles que soient sa finesse et son innocence, ne fait pas le poids devant la réalité brute. Aussi le produit du tournage, peu importe les falsifications qu'il recèle, apparaît-il minimalement comme un moyen d'objectivation, à tel point qu'il se substitue parfois avantageusement au témoignage direct des événements. Cette propriété du médium, Perrault la reconnaît volontiers et essaie d'en tirer le meilleur parti :

\begin{abstract}
Martin tourne en mon absence. Ce que je vois au retour, je ne le voyais pas de la même façon, du même angle, à travers mes jumelles qui composent avec la lumière autrement que les lentilles. Mémoire de ce que je n'ai pas vu. Et j'en parle comme si j'y étais. Force de l'image. J'en parle dans l'image. En vérité. Limage de ce que je n'ai pas vu est-elle plus vraie que la mémoire de ce que j'ai vu de loin, autrement? Sans doute.
\end{abstract}

Pour isoler les effets de la "désignation" cinématographique, il suffit, en faisant abstraction du commentaire, de circonscrire les éléments de signification géographiquement et historiquement vérifiables, ces éléments qui nous permettraient de dire que c'est $i c i$, dans cet angle, avec telle lentille, en visant ce bœuf- $c i$, à $c e$ moment précis du jour et de la saison, dans telles conditions 
climatiques que s'est effectuée la prise de vue. En d'autres termes, de par sa seule présence, la lumière constitue la preuve de l'existence de quelque chose, elle qui d'aucune manière, même dans le cas du film de fiction, ne saurait générer la matière: en deçà de sa mise en discours, il y a la contingence de ce qui se trouve audevant de la lentille, carton, papier pâte, maquette, dessin, décor, acteurs ou réalité, même si tout concourt par la suite à leur donner l'allure d'objets intentionnels.

La "désignation", ou constatation en images, ne suffit toutefois pas à raconter une histoire ou à rendre compte du réel. Se limiter à ce seul procédé n'a d'ailleurs pas de sens, tout comme écrire un livre qui se proposerait de tout dire par la description. Revenu de son voyage, Pierre Perrault s'émerveille assurément devant la vérité du souvenir suscité par le visionnement, mais il est conscient de tout ce qui risque de tomber irrémédiablement dans l'oubli, par défaut de s'être trouvé dans le champ de la caméra au bon moment :

L'image me rend la mémoire. Je suis sur place. Je m'attends à tout. A plus encore. Comme si quelque chose pouvait arriver sur l'écran. Quelque chose de nouveau. Que nous n'avons pas filmé. Une suite. Mais l'image impitoyablement s'arrête au bout du rouleau. Nous sommes toujours au bout du rouleau et insatiables! On pourrait mieux dire! Dire plus!

La perte d'information est de taille, la déception encore plus grande ; c'est pourquoi l'entreprise de "monstration" du bœuf musqué, aussi objective et documentaire fût-elle, ne se suffit jamais à elle-même :

Il faudrait pouvoir s'arrêter longuement. Fixer le mouvement. Abolir le temps qui passe. Immobiliser la vie. Mémoriser seconde par seconde. Puis remettre en mouvement, permettre les changements, suivre chaque bête, s'amuser des veaux impénitents dans les jupes des mères inquiètes. Mémoriser aussi l'ensemble, la fresque, toute la paroi mouvementée. Monumentale.

L'omniprésence de l'image, comme ce passage le laisse entendre, en appelle à une extrapolation de la lumière, à une 
amplification de la lumière, à une condensation de la lumière que sa mise en discours et l'adjonction d'une bande sonore se montreront peut-être capables de réaliser. Mais qu'adviendra-t-il alors de l'objectivité?

Fixer un objet du regard ou l'isoler de quelque manière, c'est déjà l'opposer à tout ce qui n'est pas lui, à tout ce qui se trouve au-delà de lui, à tout ce qui lui confere un caractère d'individualité. Dans le cas qui nous intéresse, déjà reconnus comme marginaux par les sciences de la nature, les plantes, les animaux et la géographie qui nous sont montrés, que le tournage discerne dans la mouvance des événements, revêtent une expressivité d'autant plus forte que chaque spécimen, en lui-même, fait figure de curiosité. L'espace, incidemment, ne prend consistance que relativement aux déplacements du bœuf musqué, il se fond avec lui dans un élan qui abolit les dimensions et souligne l'énigmatique de la transhumance. Les bêtes ont occupé le territoire depuis des millénaires, mais personne ne sait encore avec certitude les détails de leur parcours. Peut-être est-ce la main de l'artiste qui, ici et là, a le mieux rendu l'instantanéité de sa présence en remplissant les parois des cavernes et des musées:

Bosses, cornes, œil, selles, crinières. Tout cela dans une seule masse, dans une sorte de bruissement, une frayère imagée, comme dans les peintures pariétales de PechMerle, de Lascaux, comme sur le plafond peint d'Altamira où les formes sont dessinées de bêtes, de cornes, de face, de profils, d'hommes, d'arcs, de lances, de signes, de cabales, de barbelés [...] en superposition, en surimpression, comme en stratigraphie, comme en enfantillage, qui s'amusent sur la même ardoise à accumuler, attroupant, conjuguant, signifiant, empruntant têtes, croupes, naseaux, cornes, déplaçant comme pour signifier l'innombrable les angles et les postures, enchevêtrant les mouvements, les directions, les âges, les postures afin de signifier l'insignifiant, de dire l'indicible, de décrire l'indescriptible, de vérifier l'invraisemblable, de donner à entendre l'inouï, de mémoriser l'inoubliable.

Devant la force du procédé, Perrault prolonge avec des mots la simple désignation de ce qu'il voit parce que la parole lui 
semble apte, pour ne pas dire indispensable à l'élucidation de l'Oumigmag. Sauf que cette fois, contrairement au chasseur au tambour, il laissera un nom sur la paroi des cavernes. Il crée de nouvelles figures, imprécises à l'instar du réel, sans cesser de s'inspirer de ce qu'il y a d'indubitable dans la lumière; ce faisant, il s'adonne à une poésie du "phénomène", à une poésie évidemment spéculative à bien des égards, dont nous sommes appelés à juger par confrontation avec l'image. Ainsi se superposent des données en apparence contradictoires du film, la désignation visuelle et l'interprétation verbale ${ }^{15}$. Le sont-elles autant qu'on pourrait le croire?

Puis tout à coup, plus de bœuf musqué. Seulement des empreintes et des signes qui, faute de mieux, portent le regard sur ce qui ne relève pas de lui. C'est dans l'absence que ressurgit la cardinalité et avec elle le lyrisme d'une dénomination qui, manifestement, se trouve dans le prolongement de l'œuvre du frère Marie-Victorin. Tout est maintenant si facile: les fleurs ont un nom qui parle, qui nous révèle les splendeurs de l'éphémère; les animaux ont le leur, qui nous en dit tout autant sur ce qu'ils font là, à proximité des glaciers. La saxifrage, les salicaires, l'épilobe boréale, le saule, les lichens de bure, l'oseille, la pédiculaire, l'anémone; la perdrix des neiges, l'aigle, le caribou, la belette, le labbe à longue queue, tant de vie qui fixe la longitude et la latitude, que le biologiste associe à ce lieu et qui disparaît en présence de la harde. Pourtant, aux yeux d'un petit nombre de spécialistes, l'Oumigmag possède aussi un pedigree; sauf que le nom "bœuf musqué" ne rend aucunement justice à sa formidable persistance, à ses cornouailles, à sa logique instinctive, à sa prudence filiale. Le lexique d'une autre langue, semble-t-il, en a conservé le secret, qui ne demande qu'à rejaillir à la mention du nom. Il y a là une hypothèse qui convie l'interprète à en rechercher le sens derrière l'évidence.

Résumons ce que nous venons d'établir. Des sensations enregistrées sur pellicule se distinguent dans un ensemble complexe de signes, celui de la Baie-aux-Feuilles. À partir du moment où elles sont captées, offrant la part d'objectivité que permet la désignation, elles s'opposent nécessairement à autre chose, le non-filmé, et deviennent par le fait même singulières. $\mathrm{Si}$, en 
plus, elles se réferent à un objet vers quoi tout converge, il y a fort à parier que celui-ci recevra une appellation distinctive. Cependant, Pierre Perrault arrive clairement à la conclusion que la "monstration" n'est pas une fin en soi et qu'elle ne recouvre pas toutes les nuances de la dénomination:

La trace de la lumière sur la pellicule est indubitable. Est-elle lisible? Cette transparence est opaque. Elle ne va pas toute seule. Elle ne dit pas tout. Et pourtant elle accumule les informations incontestables. Comment ne pas intervenir? L'objectivité ne suffit pas peut-être à tout dire. L'objectivité garde peut-être le silence sur l'essentiel ? L'objectivité comme une objection. La subjectivité comme une relation.

Pour nommer correctement le réel, ne faut-il pas que le chasseur d'images attribue un sens à sa capture, qu'il aille au-delà de l'enregistrement du phénomène, en un mot qu'il raconte son histoire?

Nous avons retrouvé l'image de la bête dans le secret des cavernes. Mais que savons-nous de la pensée du chasseur, de ses ruses, de ses fatigues, de ses victoires? Le physicien essaie de comprendre la matière en réfléchissant toutes les images que lui transmettent les accélérateurs. Le chasseur a mis des millénaires à comprendre la bête et à mettre au point toutes ses ruses et à inventer le propulseur avant l'arc. Que savons-nous de ce qu'il a appris de la bête et de ses méfiances?

Cela nous amène inévitablement à nous demander s'il existe une objectivité de la parole, une poésie qui échappe à la gouverne des préconceptions. Notre attention est captivée et manipulée par une image et par des mots qui, loin de former un calque de l'objet, indiquent au spectateur ce qu'il faut en penser. Aussi bien dire que ce qui lui est proposé se voit octroyer un certain nombre d'attributs qui peuvent être de deux types: les premiers génèrent une idée explicite, déterminée et ordonnée qui ne varie pas d'un individu à l'autre; les seconds, au contraire, s'avèrent seulement cohérents par l'intermédiaire d'un concept suggéré par le cinéaste et ne manquent pas de fluctuer en conséquence. Dans la première catégorie se regroupent autour du 
nom l'Oumigmag et de la nomenclature nordique des prédicats en quelque sorte prévisibles, en ce sens qu'ils décrivent l'aspect générique du bœuf musqué, les traits communs à tous les spécimens du troupeau, ce que la science, en bref, a jugé bon de retenir à des fins d'analyse. En cela, l'animal constitue une famille aux caractéristiques peut-être encore mal connues, mais assurément déterminées. Pour remplir cette seconde fonction, celle de la "dénotation", nous attendions de Pierre Perrault qu'il présente le paradigme de l'espèce, son invariabilité, son territoire, sa grégarité, son exode, ses ennemis naturels, par le biais de descriptions définies qui la situent dans la hiérarchie des classifications biologiques; en fait il n'en fut rien, son film évitant de déplier un catalogue des premières apparences, en supposant que l'essentiel se trouve ailleurs, dans l'individualité de chaque bête, dans ce qu'elle fait ici et maintenant dans la fulguration du moment.

De toute évidence, ce volet de la signification du nom propre fut volontairement mis de côté au profit d'autre chose, afin que l'énigmatique, ou l'" oumigmatique " selon les préférences, demeure inaltéré. Pourtant, Didier Le Henaff et Joseph Svoboda, proches collaborateurs du cinéaste et experts de la question, connaissaient bien l'animal, au point de décoder les signes d'une attaque qui aurait pu survenir en cours de tournage ainsi que les habitudes de migration du troupeau. Perrault savait, de même que tous les membres de l'expédition, que vivant dans des conditions trop difficiles sur des îles de l'Arctique, quelques spécimens robustes furent capturés puis relâchés en Ungava, dans la région de Kuujjuaq pour être plus précis, où ils se reproduisent désormais allégrement et font montre d'une grande vigueur. Vivant plus aisément au $60^{\circ}$ parallèle qu'au $80^{\circ}$, il faut croire que les connaissances de l'homme ont récemment contribué à l'extraordinaire pérennité de l'Oumigmag, comme elles le firent sans doute à un moment ou l'autre depuis qu'un chasseur inconnu en esquissa à peine le profil il y a plus de 12000 ans. Tout ceci, en quelque sorte, se trouve éludé du documentaire: cette compétence classificatoire détourne trop l'attention de l'immédiat et menace sourdement d'émousser la contemplation du réel. 
Rien d'étonnant alors à ce que tout soit mis en œuvre pour limiter l'étendue de la "dénotation". Élaborant un discours de la bête à partir d'un nombre fort limité d'images et de figures redondantes, Perrault ne livre en fait qu'une information documentaire limitée, n'insistant que très peu sur les traits susceptibles d'éclairer les propriétés constantes du bœuf musqué. Ainsi, l'animal entrevu par "désignation" et décrit uniquement par son regard, ses cornes, son front, ses pattes, sa laine et sa fuite convergente conserve une grande partie de son mystère et s'abstrait effectivement, comme le souhaitait le réalisateur, de la mouvance irréversible du temps et de l'espace. Délibérée et calculée, cette ellipse impose un autre genre de rigueur de l'objectif documentaire.

A la faiblesse de la fonction de "dénotation", cette habile stratégie concourt à opposer la richesse inouïe des métaphores "connotatives", qui ne deviennent cohérentes que sur acceptation d'un critère d'interprétation fourni par l'auteur. Ici, plus question de s'accorder à une connaissance reconnue et sanctionnée par la zoologie, mais bien au contraire d'explorer ce qu'ignorent les canons de la science en faisant germer dans l'esprit de chaque interprète une idée plausible, mais autre de la réalité. Le bœuf musqué ne signifie sans doute pas la même chose pour Martin Leclerc, cameraman, que pour le monteur Camille Laperrière et pourtant leur connaissance dénotative et désignative du nom l'Oumigmag doit sensiblement se ressembler.

Comment réagir uniformément à des figures telles "la harde versante et versatile", "le sommet du vent qui vente", "l'unanime magnificence d'une houleuse cavalcade", "les multiples sons d'un silence quaternaire", "les maximes laineuses", "les minuscules grouillements de pucerons sur le cuir précambrien", "les cornes ombrageuses et les résines de la nuit calcaire", "la lente procession poilue", "l'arrogance des crinières", "le regard par l'entremise des cornes", toutes sorties de l'imaginaire du poète et offertes en complément, sinon par anticipation du discours visuel? Que l'interprète ne s'y trompe pas: ces métaphores n'ont rien de gratuit ou de superficiel, elles visent seulement à enrichir le sens évocateur de l'Oumigmag, à révéler des aspects incompris et bien réels de sa contingence, à prodiguer une information en marge de nos a priori, à déceler une vérité inaccessible 
au regard. La fable de la découverte du monde, les mythes enseignés et acceptés depuis l'enfance, la civilisation et les livres forment-ils un écran entre nous et le bœuf musqué? Leur manière de voir et de penser agit-elle à la façon d'un filtre déformant la lumière, le grain et la perspective? Voilà une hypothèse qui n'est pas seulement le fait des insuffisances ou des orientations du tournage: par une désautomatisation des habitudes sensorielles, elle recherche l'invisible et l'indicible.

Bien entendu, la constitution des plans, les limites du champ, l'angle de vue, le montage, le commentaire, le son et la musique ne sauraient reproduire fidèlement l'evénement. Inutile de le répéter à Pierre Perrault, qui s'est prononcé sur la question. Néanmoins est-ce la véritable raison du double constat d'échec du réalisateur, comme s'il s'agissait d'un projet impossible, d'une entreprise inachevée, d'un film raté? "Ces rencontres accumulées, à l'en croire, ne font pas une circonstance à raconter. "Puis il conclut: "Nous étions à la recherche de l'Oumigmag, nous n'avons rencontré que l'énigmatique [...] le renard est plus facile à apprivoiser dans la fable que dans la réalité et il nous faudra tout recommencer."

Tout reprendre depuis le début? Certainement non, car ce beau documentaire de vingt-huit minutes, d'une façon brillante et éclatante, ne fait que constater le caractère magique de toute vélléité de dénomination. À la limite, ne pourrait-on même imaginer qu'une équipe de cinéastes parviendrait à constituer une banque mémorielle, un registre de "désignations " lumineuses qui réussirait à individualiser le bœuf musqué, à le baptiser et à le sémantiser? La dénomination étant un processus continu, il y a fort à parier que ces mêmes gens trouveraient le moyen de lui accoler une "dénotation" pratiquement exhaustive, pour peu qu'ils en voient la nécessité, ce qui aurait pour effet d'enrichir une nomenclature à la mesure du pays. En contrepartie - et Pierre Perrault s'est montré bien hardi de se lancer dans une pareille aventure - , il semble que le volet "connotatif" du nom propre, celui dont les figures de rhétorique nous donnent un aperçu inégal, ne finira jamais par se stabiliser au point de devenir objectif, c'est-à-dire "réel", au sens de rendre compte de l'indubitabilité de la lumière. 
Faut-il le déplorer? Bien au contraire, l'objectif documentaire remplit mieux son mandat lorsqu'il s'adonne à une recherche allusive. Le chasseur au tambour, le "penseur sauvage" tenaillé par la faim connaissait davantage l'Oumigmag que l'intellectuel d'aujourd'hui. Pour lui, ce nom prenait une résonance subtile, sentie et merveilleusement soutenue par l'observation, ce dont le documentaire traditionnel ne donne qu'un maigre aperçu. Le grand mérite de la manière de Pierre Perrault, c'est peut-être de démontrer la compatibilité du langage poétique avec l'objectivité puisque procédant de la sorte, il se rapproche un peu plus du savoir incommensurable du sculpteur de Laugerie. Quant au fait qu'il ait finalement abdiqué devant la complexité de son objet, s'agit-il davantage de respect et de sagesse que des déboires d'un groupe de chasseurs d'images?

Université Laval

\section{NOTES}

1 Cette affirmation s'appuie sur un texte de Pierre Perrault dont le titre provisoire est L'Oumigmatique ou l'Objectif documentaire. Hormis une publication partielle grâce aux soins de l'ONF en 1989 dans le cadre du festival Le Documentaire se fete, cette réflexion demeure en grande partie inédite. Avec la permission de l'auteur, nous le citerons régulièrement au cours de ce texte.

2 Gottlob Frege, "On Sense and Nominatum ", dans Fiegl et Sellars (direction), Readings in Philosophical Analysis, Londres: Appleton-Century, 1949.

3 John Stuart Mill, A System of Logic, Rationative and Inductive, Londres: Longmans Green \& Compagny, 1879.

4 Bertrand Russel, An Inquiry into Meaning and Truth, Londres: George Allen, 1940.

5 Ludwig Wittgenstein, Philosophycal Investigations, Oxford: Basil Blackwell, 1954.

6 Paul F. Strawson, Introduction to Logical Theory, Londres: Methuen, 1952.

7 John R. Searle, Les Actes de langage, Paris: Hermann, 1972.

8 John Algeo, On Defining the Proper Name, Gainesville: University of Florida Press, 1973.

9 Farhang Zabeeh, What is in a Name, The Hague: Martinus Nijhoff, 1968.

10 Holger Sørensen, The Meaning of Proper Names, Copenhagen: G.E.C. Gad Publisher, 1963.

11 Allan Gardiner, The Theory of Proper Names. A Controversial Essay, Londres: Oxford Press, 1954.

12 Claude Lévi-Strauss, L'Identité, Paris: Grasset, 1977. 
13 Ouvrage cité par Anna Makikin dans Name, Hero, Icon (Berlin: Mouton de Gruyter, 1992). C'est nous qui traduisons.

14 A notre connaissance, il n'y a que dans la série $A u$ pays de Neufve-France où la problématique de la dénomination du pays n'est pas abordée directement.

15 En regard de la théorie du nom propre, il existe en fait six niveaux de signification: la désignation, la dénotation et la connotation par l'image, ainsi que la désignation, la dénotation et la connotation par la parole. À l'instar de Perrault, nous insistons sur les deux extrêmes de cette typologie (soulignés ci-dessus), dans le but de montrer que l'un et l'autre peuvent figurer dans un film documentaire. 